
December 1942

\title{
COMBINATION OF WOOL PROTEIN WITH ACIDS IN MIX- TURES, AND ITS RELATION TO THE ACID DYEING OF $\mathrm{WOOL}^{1}$
}

\author{
By Jacinto Steinhardt, Charles H. Fugitt, and Milton Harris ${ }^{2}$
}

\section{ABSTRACT}

In order to extend previously reported analyses of the combination of acids with wool, measurements have been made over a wide range of concentrations, of the amounts of the anions of two acids combined by wool fibers when acids of different affinity for protein are present in different proportions or in mixtures of their acid and salt forms. It is shown that anions compete with one another for combination with the fiber, so that the amounts of each combined depend not only on the amounts initially present but also on their respective affinities for wool. The bearing of these results and their interpretation on the theory of acid dyeing is discussed, with special reference to the factors promoting the attainment of "level" or "solid" application of dye to the fibers

\section{CONTENTS}

I. Introduction

II. Experimental procedure

III. Results and discussion _...

IV. Relation of acidity and anion affinity to level dyeing $\ldots$

V. References

\section{INTRODUCTION}

Earlier papers have demonstrated that the tendency of wool to combine with different strong acids differs widely and have discussed the bearing of these differences on the theory of dying $[6,7] .^{3}$ The differences were interpreted in terms of the combination of wool with anions as well as hydrogen ions, the combination with each anion being governed by its own distinct affinity. ${ }^{4}$ Numerical values of these affinities (expressed as reciprocals of dissociation constants), for 33 strong acids, have been tabulated elsewhere [7].

The earlier work, which was limited to studies of individual acids, has now been extended to include an investigation of the combination of wool with acids present in mixtures. The new experiments contribute to an understanding of the more complex equilibria which obtain in mixtures [3], and should be more pertinent to the process of

\footnotetext{
1 A brief account of this work appeared in Proc. Am. Soc. Biological Chemists XXXV, J. Biol. Chem. 140, $\operatorname{cxxiv}(1941)$.

2 Research Associates at the National Bureau of Standards, representing the Textile Foundation.

3 Figures in brackets indicate the literature references at the end of this paper.

4 The use of the term "affinity" in discussions of dyeing is often ambiguous, because two different meanings have been attached to it. In the present paper the term denotes the extent to which, under equilibrium conditions, the ion or acid is associated with the protein. It is not used, as in commercial practice, to describe the rapidity with which dyes combine with the fiber.
} 
dyeing in complicated baths than were the simpler experiments. The results also contribute to an understanding of the pari played in the dyeing process by acids, and by such commonly used assistants as Glauber's salt.

\section{EXPERIMENTAL PROCEDURE}

Methods of purification of the wool, measurement and interpretation of $\mathrm{pH}$ values, determination of the amounts of acid bound, and application of corrections for the effects of hydrolytic decomposition of the fibers at high temperatures and high acidities have been described in earlier publications $[4,5,7]$.

Each of a number of samples of wool, conditioned at $21^{\circ} \mathrm{C}$ and 65 percent relative humidity, was immersed in about 80 times its weight of solution. The solutions contained either the acid form of the dye, Orange II, or its sodium salt. In addition, each solution contained a second acid, either hydrochloric or naphthalene- $\beta$-sulfonic acid. The concentrations of the dye varied between 0.0004 and $0.025 M$, while the second acid was present either in equimolar amounts or in amounts 10 times as great.

The solutions were kept at $50^{\circ} \pm 0.1^{\circ} \mathrm{C}$ for from 3 to 5 days, periods which have been shown in earlier work $[5,7]$ to be sufficient for a close approach to equilibrium. The following measurements were then made on aliquots of each: (a) $\mathrm{pH}$ at room temperature; (b) total concentration of acid by titration; (c) dye concentration, colorimetrically; (d) ammonia produced as the result of hydrolytic decomposition; and (e) in some of the experiments with hydrochloric acid, concentration of chloride by the Volhard method, after decolorizing the solution with hydrogen peroxide. When only acids were present, the amounts of chloride combined with the fiber, calculated from the results of analyses, agreed closely with the amounts calculated by subtracting the amount of dye combined from the total acid combined. Advantage was taken of this agreement to calculate the amounts of naphthalenesulfonic acid, since no method for readily and directly estimating the concentration of this ion is available.

\section{RESULTS AND DISCUSSION}

The experiments to be described fall into three categories: (1) Measurements obtained with mixtures of two acids inititally present in equimolar proportions, (2) measurements obtained with mixtures of two acids in initially fixed but unequal proportions, and (3) measurements obtained with mixtures of a salt of one acid in equimolar proportion with the second acid. Data from each group of experiments are represented graphically in figures 1,2 , and 3 , respectively. ${ }^{5}$

It will be observed that in the experiments with two acids (figs. 1 and 2 ) both the total acid and the dye anion combined increase steadily as the acidity increases, while the amount of the second anion combined increases at first, but then diminishes sharply. The maximum amounts of the second anion combined, and the $\mathrm{pH}$ values at which these maxima are found, differ in each experiment; they depend not only on the concentration ratio of the two acids but on the specific properties of the second acid as well. Thus, the maximum amount of naphtha-

\footnotetext{
5 The data on which these figures are based are tabulated elsewhere [3].
} 
lenesulfonate combined is considerably larger than the amount of chloride. This is in keeping with the relative affinities for wool of these two anions, as previously reported [6]. Of the two anions which compete with dye, the large naphthalenesulfonate anion competes far more effectively than the smaller chloride ion. The broken lines that follow the course of the curve representing combination with the second anion in certain of the figures have been computed from the respective affinities of the ions involved, as is explained elsewhere [3].

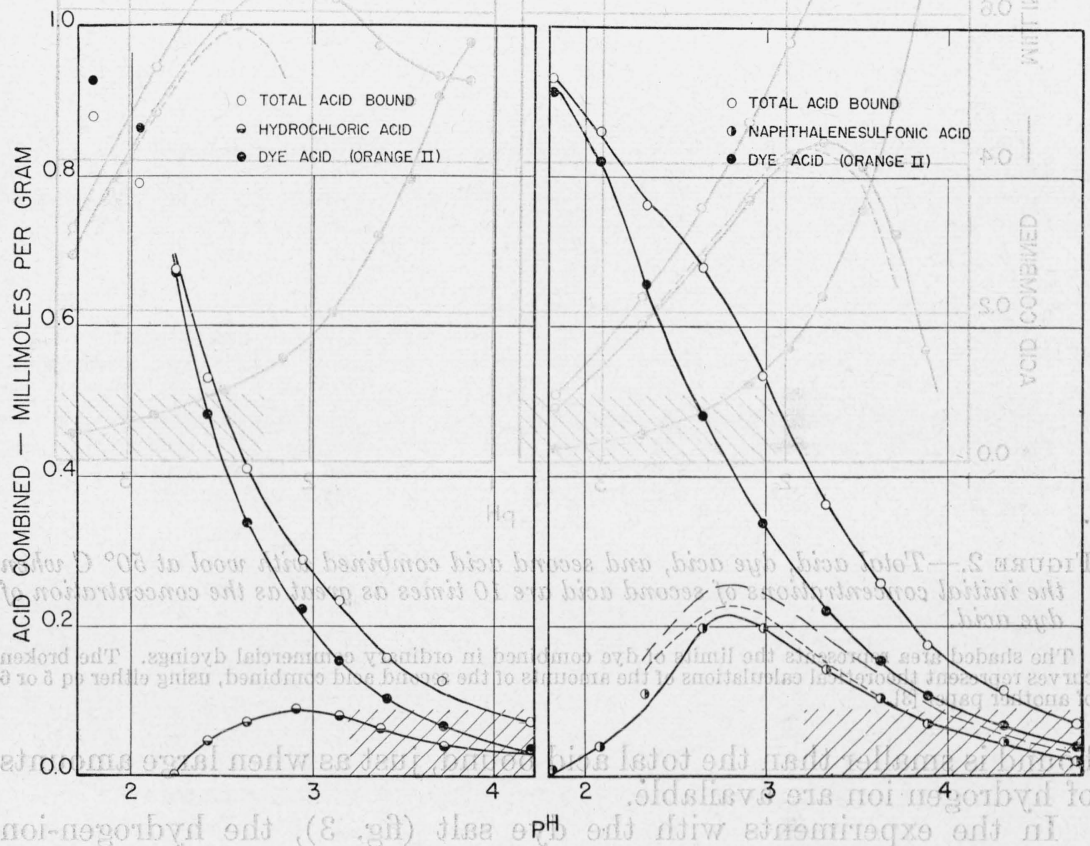

Figure 1.- Total acid, dye acid, and second acid combined with wool at $50^{\circ} \mathrm{C}$ when the dye acid and second acid are initially present in equimolar proportions.

The shaded area represents the limits of dye combined in ordinary commercial dyeings. The broken curves represent theoretical calculations for the combination of naphthalenesulfonic acid. The upper curve has been obtained by the method of eq 4 in another paper [3], and the lower curve represents both eq 5 and 6 of the same paper.

In the experiments with the dye salt ([3], and fig. 3), the more limited supply of hydrogen ion results in the combination of smaller amounts of acid at a given concentration of dye anion than in the other two sets of experiments. Distinctions due to the difference in affinity of the anions are greatly accentuated; thus with hydrochloric acid no detectable amount of the second anion (chloride) combines with the wool. ${ }^{6}$ Indeed, the affinity of the dye anion is great enough to result in a combination of slightly more dye anion than of hydrogen ion when the supply of the latter is limited. However, when the second anion has a sufficiently high affinity to compete effectively with the dye even under these more severe conditions, the amount of dye

6 The results obtained with mixtures of an acid and a salt depend on maintaining exact equivalence between the amounts of each initially present. The resemblance to the results obtained with acids alone is diminished if this equivalence is not maintained. The extent of this effect is indicated by a single point in figure 3, obtained with an initial amount of acid 3 percent in excess of the amount of dye. 


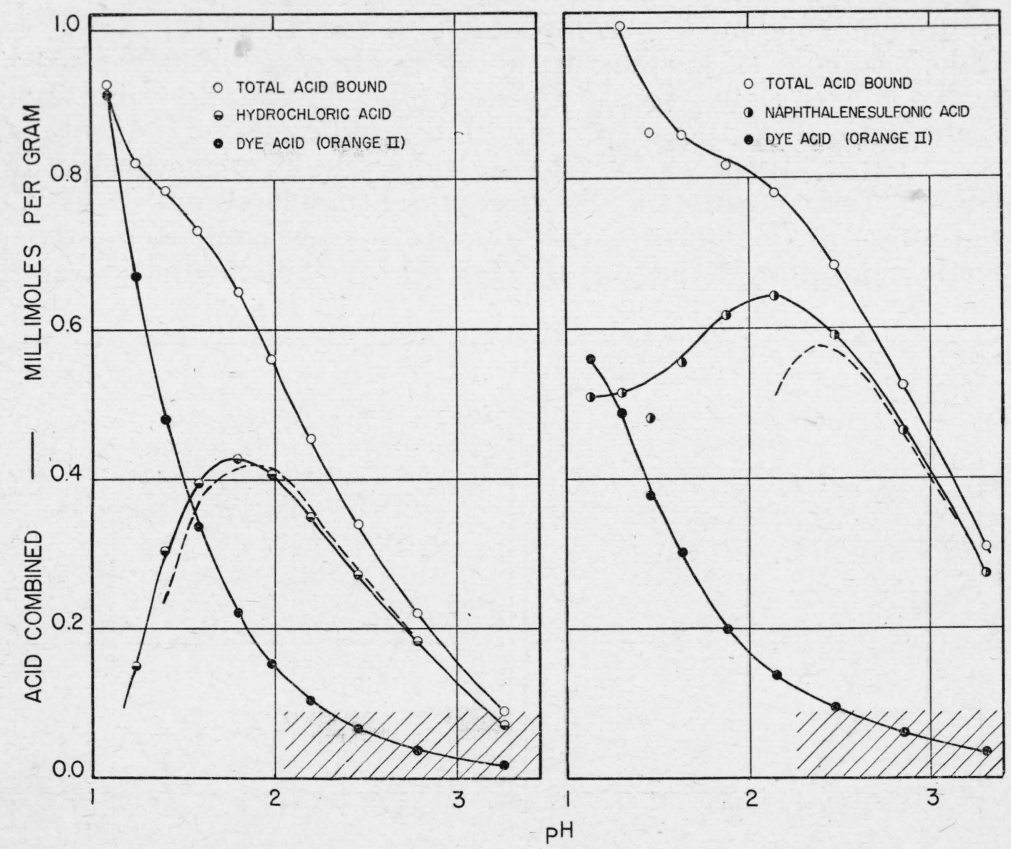

Figure 2.-Total acid, dye acid, and second acid combined with wool at $50^{\circ} \mathrm{C}$ when the initial concentrations of second acid are 10 times as great as the concentration of dye acid.

The shaded area represents the limits of dye combined in ordinary commercial dyeings. The broken curves represent theoretical calculations of the amounts of the second acid combined, using either eq 5 or 6 of another paper [3].

bound is smaller than the total acid bound, just as when large amounts of hydrogen ion are available.

In the experiments with the dye salt (fig. 3), the hydrogen-ion concentration and the dye-anion concentration were initially equal, as in experiments with the dye acid alone [6, 7]. However, these initially equal concentrations of hydrogen ion and dye anion are here not necessarily equal at equilibrium, and small differences between these results and those previously reported are to be expected.

Differences in the initial ratio of hydrogen-ion concentration to concentration of the colored anion of the dye, which distinguish each set of experiments (figs. 1, 2, and 3), result in marked differences in the degree of exhaustion of the dye from the solution onto the fibers. The higher is this ratio, the greater is the degree of exhaustion found. Since more acid also remains in the bath at equilibrium, the higher ratios also result in a displacement of the curve of combination of the dye acid in the direction of lower $\mathrm{pH}$ values.

However, neither the degree of exhaustion of the dye nor the equilibrium $\mathrm{pH}$ obtained with a given initial concentration of dye depends solely upon the ratio of acid to dye. With the same ratio of concentrations of the two anions, a higher degree of dye exhaustion is obtained with hydrochloric acid than with naphthalene- $\beta$-sulfonic acid. Thus, for example, in the presence of hydrochloric acid, 10.3 percent of an initially $0.01 M$ dye solution remains uncombined. 


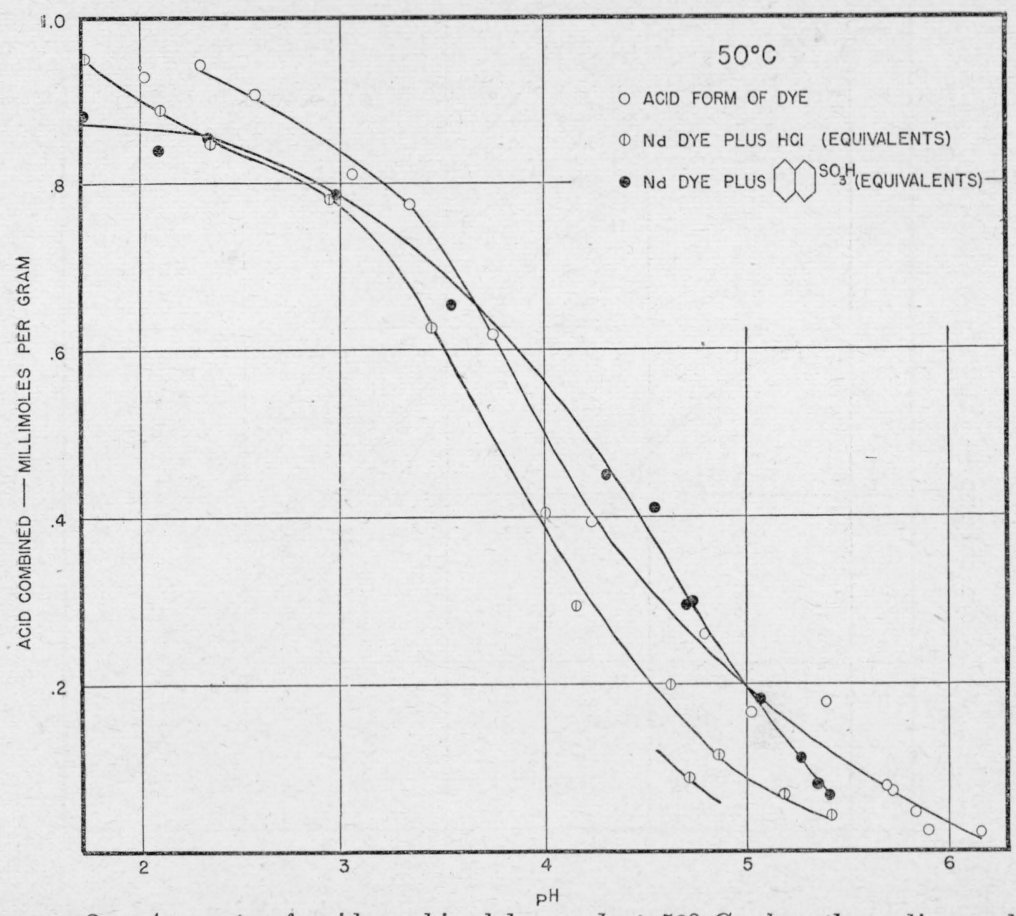

Figure 3.-Amounts of acid combined by wool at $50^{\circ} \mathrm{C}$ when the sodium salt of Orange II is added in equivalent amounts to either hydrochloric or naphthalene- $\beta$ sulfonic acid.

The amount of dye acid combined when the acid form of Orange II alone is present is also ropresented. The significance of the single point which lies on a separate curve is explained in footnote 6 .

When naphthalenesulfonic acid is used, the corresponding figure is 15.1 percent. Comparable differences appear in the experiments with the dye salt. Even greater differences appear in the experiments with a large excess of acid (fig. 2), where 3.3 percent of the dye remains uncombined in the presence of hydrochloric acid while a much larger amount, 35.3 percent, remains uncombined with the same initial amounts of naphthalenesulfonic acid. Differences in the degree of exhaustion of the dye are found even at the same $\mathrm{pH}$ and therefore cannot be accounted for in terms of the promotion of dye combination which is brought about by hydrogen ions.

The large differences in the amounts of dye remaining uncombined in the presence of different second anions can be shown by plotting the amounts combined against the negative of the logarithm of the dye concentration at equilibrium. This is done in figure 4, in which has been included for comparison data previously published [7] for Orange II alone. As the figure shows, in the presence of a large excess of naphthalenesulfonic acid the quantity of dye uncombined, although small, is approximately 100 times greater than when the same excess of hydrochloric acid is present. Similar but smaller differences appear in the presence of lower concentrations of the second acid. It may be shown that differences such as these are predictable consequences of the anion competition postulated [3].

$491983-42-3$ 


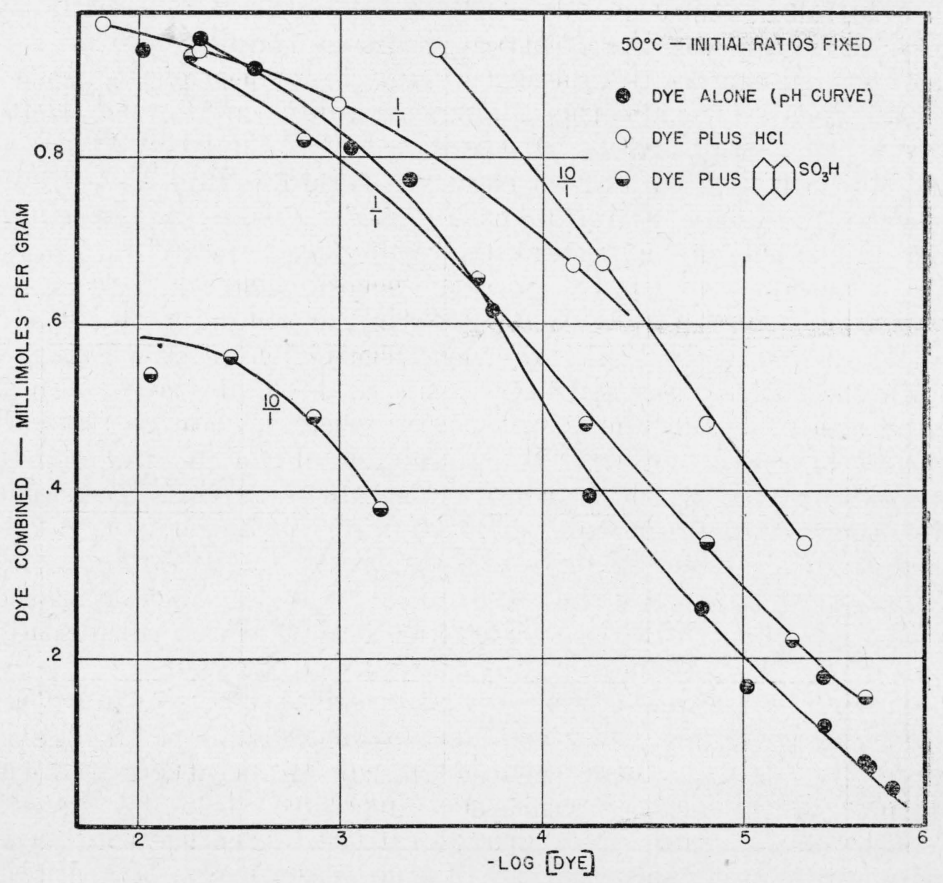

FIGURE 4.-Comparison of the amounts of dye combined by wool at $50^{\circ} \mathrm{C}$ at different equilibrium concentrations of dye (moles per liter) when the dye acid is used alone, and when other acids are present in various ratios.

\section{RELATION OF ACIDITY AND ANION AFFINITY TO LEVEL DYEING}

The behavior of wool in acid dyeing is conditioned by the fact that it contains acidic and basic groups, and by the fact that the dye ions commonly involved are anions. Although the anions taken up by the fibers are probably held at or near the $\mathrm{RNH}_{3}^{+}$, groups, it need not be maintained that dye would not be combined at all except as the result of this coulombic interaction, or as the result of an acid-basic reaction. In the absence of these factors, dyeing would still be possible since a part of the forces making for the adsorption of the large dye anions would still be exerted. The fiber would acquire a negative charge, and some of the cations present would then be electrostatically bound. ${ }^{7}$ However, dyeing behavior would be greatly altered in its relation to concentration of dye, and $\mathrm{pH}$.

The effect of hydrogen ion on the combination of dyes is distinct from that of other common cations, because of the presence of weak acidic and basic groups in wool. Unlike such ions as sodium, hydrogen ion can form a covalent bond with oxygen at carboxylate groups, and is therefore bound to a greater extent. Thus, the addition of hydrogen

${ }^{7}$ Although the forces binding the anion to the fiber may be only in part ionic (coulombic), as is shown by the wide difference in the energy of binding of ions of low and of high affinity [6,7], the contribution of the ionic energy is very important. The latter exerts an influence over sufficiently great distances to affect the distribution of the ions in solution in a manner favorable to the interaction of the short-range (van der Waal's) forces. These forces operate more effectively when the ions approach the fiber closely under the influence of longer range (coulombic) forces. In the absence of the latter, the amount of noncoulombic association possible would be much reduced, and with the smaller ions, might be entirely negligible. 
ion to a solution containing a dye will tend to promote the exhaustion of the dye by increasing the positive charge on the fiber.

It has been shown by the measurements described in the preceding pages that, besides the hydrogen ion, the anion introduced with the acid affects the equilibrium between the wool and the dye. The effect of the addition of acid is clearly twofold. The hydrogen ion increases the tendency of dye to combine with wool by lowering the $\mathrm{pH}$, just as in all the experiments previously reported. There is, however, a tendency of the anion of the second acid itself to combine with the wool. By thus competing with the anion of the dye, the anion of the second acid reduces the increased exhaust which is due to the addition of excess hydrogen ion. Indeed, if the tendency of the second anion to combine is sufficiently great, its competitive effect may nullify and even reverse the tendency of the hydrogen ion to promote exhaustion of the dye onto the fibers. With hydrochloric acid the exhaustion-promoting effect of the hydrogen ion far outweighs the competitive effect of the chloride ion. With naphthalenesulfonic acid in excess the opposite is true: combination of the wool with its anion occurs to so great an extent that combination of the fibers with the dye anion is greatly reduced (fig. 4).8

Anions other than that of the dye may affect the results obtained in acid dyeing in another way. When the acid form of Orange II is used alone $[6,7]$, the fibers always appear to be dyed uniformly. Microscopic examination of cross sections show that the fibers are dyed "solidly" (i. e., uniformly throughout their cross section) as well. The same uniformity and solidity of dye distribution are obtained when either high or low concentrations of naphthalenesulfonic acid are also present. However, when hydrochloric acid is used, the fibers are dyed very unevenly and lack solidity. In general, an unlevel dyeing usually results under conditions which lead to combination with a very high percentage of the dye initially present in the bath, that is, to a high degree of exhaust.

If it is considered that even carefully selected and purified fibers, such as those used in the present work, lack uniformity, not only with respect to size of cross section but also with respect to previous history, the correlation between unlevel dyeing and high exhaust may be understood. Differences between fibers, or parts of fibers, are known to result in large differences in the rates at which dye is taken up. Under conditions favorable to a high degree of exhaust, the parts of the fibers which dye quickly are soon heavily dyed, and other parts of the fibers can only be lightly dyed or are not dyed at all. At equilibrium, the dye would probably be distributed uniformly, but the rate at which a redistribution may take place is greatly limited by the low concentration of dye in the bath, and by the extremely low tendency for dye to dissociate from the more highly dyed portions. By keeping the hydrogen-ion concentration low, or by providing large quantities of a second anion that reduces the extent of combination of the dye, one may increase the tendency for heavily dyed fibers to dissociate dye into the solution. Redistribution of the dye can then occur relatively rapidly.

If this interpretation is correct, if follows that level dyeing will, in general, be promoted by the presence of colorless anions having a

8 Similar competitive effects have been demonstrated in the effect of various salts on the adsorption of acids by charconl [1]. 
relatively high affinity for the fiber, and which therefore compete effectively with the dye. The sulfate ion, which is usually added to dyebaths in the form of Glauber's salt, must be expected to have the leveling effect just described. The affinity of sulfate for wool is only slightly lower than that of naphthalenesulfonate and is very much greater than that of chloride. Nevertheless, work as yet unpublished has shown that naphthalenesulfonate presents some advantages over suliate as a leveling anion in the acid dyeing of wool, especially when dyes are employed with which it is difficult to achieve level or solid effects. Although many factors enter into the choice of a leveling agent, such as effectiveness in wetting, penetration of fabrics, dispersing of the dye, and cost, the present studies indicate that the best choice aside from these factors may depend on the affinity of the dye employed; with dyes of relatively low affinity, no better competition is needed than that now afforded by the addition of Glauber's salt. Indeed, too effective competition by anions of high affinity would lead to resistance to dyeing, uneconomic exhausts, and a reduction of the wash-fastness of the dye. However, with dyes of greater affinity, leveling agents capable of more effective competition that that of Glauber's salt should be expected to improve dyeing, especially of blends of different wools; they should not reduce wash-iastness if they they can be effectively removed by a cold-water rinse. Previous studies [7] furnish a basis for the selection of such leveling agents.

\section{REFERENCES}

[1] C. W. Carr, H. Freundlich, and K. Sollner, J. Am. Chem. Soc. 63, 693 (1941).

[2] J. Steinhardt, J. Research NBS \%8, 191 (1942) RP1452.

[3] J. Steinhardt, J. Research NBS \%9, 425 (1942) RP1511.

[4] J. Steinhardt and M. Harris, J. Research NBS 4, 335 (1941) RP1286; Textile Research 10, 181 (1940); Am. Dyestuff Reptr. 29, 103 (1940).

[5] J. Steinhardt, C. H. Fugitt, and M. Harris, J. Researeh NBS 25, 519 (1940) RP1343; Textile Research 11, 72 (1940); Am. Dyestuff Reptr. 29, 607 (1940).

[6] J. Steinhardt, C. H. Fugitt, and M. Harris, J. Research NBS 26, 293 (1941) RP1377; Textile Research 11, 259 (1941); Am. Dyestuff Reptr. 30, 223 (1941); J. Steinhardt, Ann. N.Y. Acad. Sci. 11, 287 (1941).

[7] J. Steinhardt, C. H. Fugitt, and M. Harris, J. Research NBS 28, 201 (1942) RP1453; Am. Dyestuff Reptr. 31, 77 (1942).

WASHINGTON, June 4, 1942. 